\title{
Geodetic base for the construction of ground- based facilities in a tropical climate
}

\author{
Anton Nikonov ${ }^{1, *}$, Nikolay Kosarev ${ }^{1}$, Olga Solnyshkova ${ }^{2}$, and Inna Makarikhina ${ }^{2}$ \\ ${ }^{1}$ Siberian State University of Geosystems and Technologies, Plakhotnogo str., 10, 630000, \\ Novosibirsk, Russia \\ ${ }^{2}$ Novosibirsk State University of Architecture and Civil Engineering (SIBSTRIN), Turgeneva str., \\ 159, 630008, Novosibirsk, Russia
}

\begin{abstract}
The data on the geodetic base creation for surveys and construction on the example of works to expand the power station capacity, performed in one of the Latin America countries in 2015-2018, are presented in the paper. The points' coordinates obtained by two methods were established. A comparison of the points' heights obtained from II class geometric leveling and two-sided trigonometric leveling is also carried out. It was concluded that the high accuracy of determining the points' coordinates and altitudes can be obtained by ground methods (using a total station) with careful measurements even in tropical climates.
\end{abstract}

\section{Introduction}

Industrial enterprises (factories, steel plants, thermal power plants), as a rule, occupy large areas (more than $1 \mathrm{~km} 2$ ). Geodetic center basis (GC) is created in order to transfer the project in nature and geodetic support for the construction of such objects. Traditionally, the center base was made in the form of a construction grid at the construction site, i.e. geodetic points were located regularly at the tops of squares with sides from 50 to $200 \mathrm{~m}$. All angles and distances were measured in this construction. The form of the network made it possible to perform centering works using the simplest tools (tape measure and theodolite). Also, microtriangulation, trilateration, and polygonometry methods were used in the process of GCB creating. Angular measurements had to be performed with an accuracy of 2-5", and linear measurements were with a relative error of no worse than 1 : 25.000 .

The accuracy of GCB construction depends on the location compliance of buildings, structures, underground utilities with the master plan, as well as the construction works' quality, in particular the assembly of frame industrial buildings. Currently, GBC is created by building linear-angle networks, using GNSS technology, and also by combining these methods.

\footnotetext{
${ }^{*}$ Corresponding author: michmacha@mail.ru
} 


\section{Materials and Methods}

It was decided to expand the existing capacity of the thermal power plant by building a new power unit on the territory of one of the Latin America countries. 36 geodetic points were laid on the power plant's territory ( 23 pylons and 13 marks were built in concrete surfaces, fig. 1) in order to provide engineering surveys, as well as work on the further power unit construction.
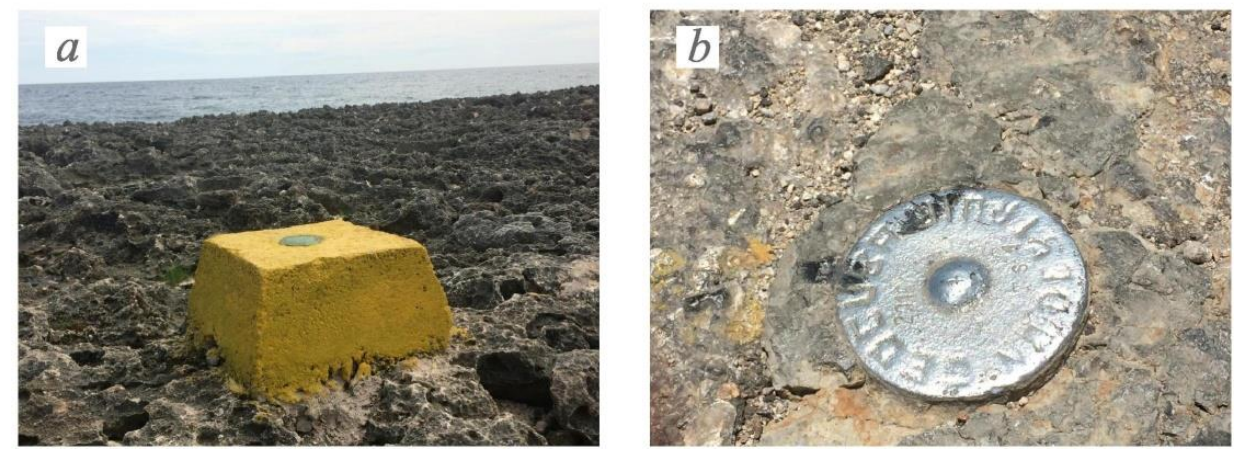

Fig. 1. Geodetic points in the form of a pylon (a) and marks in asphalt (b).

In 2017, 11 additional geodetic points (GGP) were installed, arranged in pairs (T1 T11). The newly laid points coordinates were determined on September 12.2017 by satellite method with respect to two points of the geodetic network $\mathrm{p} 1$ and $\mathrm{p} 2$. The measurements were performed using two Leica GS 10 mobile GNSS receivers in fast static mode (the observation time was about 1.5 hours). The passport accuracy of the device during the baselines measuring in the plan for this type of receivers was $3 \mathrm{~mm}+0.5 \mathrm{ppm}$.

The distances from the initial point $\mathrm{p} 1$ to the determined ones $(\mathrm{T} 1-\mathrm{T} 11)$ ranged from 39 to $41 \mathrm{~km}$. The baselines lengths, measured from point $\mathrm{p} 2$, did not exceed $15 \mathrm{~km}$. The distance between the starting points of the state base p1 and p2 was approximately $25 \mathrm{~km}$.

Fixed solutions were obtained during all baselines processing. The maximum mean square error in the plan was $13 \mathrm{~mm}$, and $26 \mathrm{~mm}$ one was in height. The residuals in all closed figures did not exceed their theoretically calculated values.

The coordinates difference of the determined points ( $\mathrm{T} 1-\mathrm{T} 11)$, obtained with respect to $\mathrm{p} 1$ and $\mathrm{p} 2$, averages $157 \mathrm{~mm}$ along $\mathrm{Y}$ axis, and $4 \mathrm{~mm}$ along $\mathrm{X}$ axis. This indicates a source data error, i.e. shows the discrepancy between $\mathrm{Y}$ coordinates of $\mathrm{p} 1$ and $\mathrm{p} 2$. As the final coordinates of $\mathrm{T} 1-\mathrm{T} 11$ points, a contractor accepted the values obtained relative to the source point $\mathrm{p} 2$ which was the closest to the work place. Thus, the coordinates of T1 - T11 points were determined by the ray method with respect to one starting point (p2), and the measurements with respect to $\mathrm{p} 1$ were for reference only.

T1 - T11 points' heights were determined from the geometric leveling of class II (allowable discrepancy was $5 \sqrt{ } L$, where $L$ was the stroke length in $\mathrm{km}$ ). Excesses were measured with a Leica LS-15 digital level with a passport accuracy of $0.3 \mathrm{~mm}$ per kilometer of double stroke.

The task was to monitor the mark's quality of 11 geodetic points and selectively to assess the accuracy of their relative position. In order to do it, it was decided to pave a closed tacheometry stroke on the newly laid and already existing geodetic points. Leica TS02 electronic total station $\left(\sigma=5^{\prime \prime}\right)$ and a standard mini-reflector were used to perform the work. The stroke was laid from 23 to 24 June 2018, in clear weather (the sun was at its zenith), the air temperature was $+30^{\circ}-+33^{\circ} \mathrm{C}$. A geodetic umbrella was not used in measurements. The work area was flat 
Each point measurements were performed in the following sequence: four pointing to the backsight with a circle left (CL), then four pointing with a circle to the right (CR), then an assistant with a stick moved to the front point and the measurements were performed in a similar pattern. So, the expected mean square error of the horizontal angle measurement would be $\sigma \approx 3^{\prime \prime}$.

The total station height above the point was measured with a laser tape measure with an accuracy of $1 \mathrm{~mm}$. The instrument was centered over a point using a laser reticle with an error of no more than $1 \mathrm{~mm}$. The error in the direction caused by inaccuracy of centering, for a minimum side length of $S_{\min }=111 \mathrm{~m}$, will not exceed $1.8^{\prime \prime}$.

\section{Results}

The tacheometric stroke is a closed polygon of $n=14$ points $2.5 \mathrm{~km}$ long (fig. 3 ).

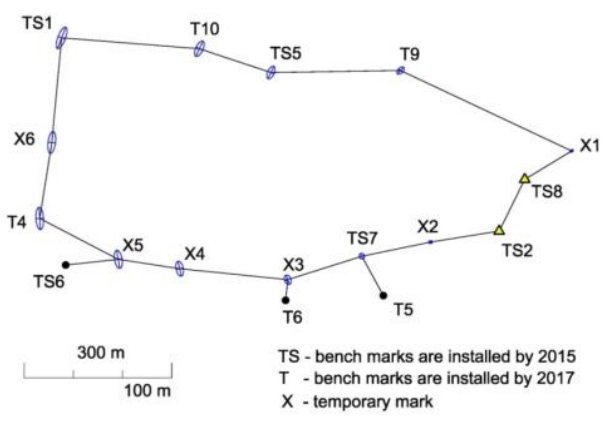

Fig. 2. Tacheometric stroke sheme.

Allowable residual is calculated as

$$
f_{\text {lim }}=2 m_{\beta} \sqrt{n}=2 \cdot 3^{\prime \prime} \cdot \sqrt{14}=22^{\prime \prime}
$$

The actual angular discrepancy was $\mathrm{f}=10^{\prime \prime}$, which did not exceed the permissible value. Technical characteristics of the tacheometric stroke are presented in table 1.

Table 1. Characteristics of the tacheometric stroke.

\begin{tabular}{|l|c|c|c|}
\hline \multicolumn{1}{|c|}{ Parameter } & Minimum & Maximum & Average \\
\hline Stroke sides' length, m & 111.3 & 384.1 & 189.2 \\
\hline $\begin{array}{l}\text { The difference of distances' average } \\
\text { values straight / back, mm }\end{array}$ & 0.3 & 2.9 & 1.2 \\
\hline SD measurements of the sides, mm & 0.23 & 1.50 & 0.91 \\
\hline $\begin{array}{l}\text { Excess difference } h \\
\text { straight / back, mm }\end{array}$ & $\begin{array}{c}1.6 \\
(S=153.2 \mathrm{M})\end{array}$ & $\begin{array}{c}68.4 \\
(S=384.1 \mathrm{M})\end{array}$ & 11.4 \\
\hline $\begin{array}{l}\text { The difference between four counts } \\
\text { in the horizontal circle CL / CR }\end{array}$ & $1.3^{\prime \prime} / 1.6^{\prime \prime}$ & $9.8^{\prime \prime} / 9.0^{\prime \prime}$ & $4.8^{\prime \prime} / 4.4^{\prime \prime}$ \\
\hline $\begin{array}{l}\text { SD position of a point in a plan of } \\
(1 \sigma), \text { mm }\end{array}$ & 1.7 & 12.5 & 7.8 \\
\hline
\end{tabular}

TS2 and TS8, laid in 2015, were taken as the baseline. The network was equalized as a free one. The mean square error of the weakest point of the tacheometric stroke (TS1) was 
$12.5 \mathrm{~mm}$, which indicated a high accuracy of the paved stroke as it followed from the table 1.

TS08 was taken as the starting point for elevation. The remaining points' heights were determined from non-simultaneous two-sided trigonometric leveling. The height of the reflector was $0.7-1.3 \mathrm{~m}$. The discrepancy of the landfill, calculated from the average elevations, was minus $6.3 \mathrm{~mm}$, which did not exceed the tolerance for level III class $(10 \sqrt{L}$, where $L$ is the stroke length in $\mathrm{km}$ ). The largest difference in elevations from measurements straight / back was $-68.4 \mathrm{~mm}$, which corresponded to the refractive index $\mathrm{k}=-2.15$ at a distance of $\mathrm{S}=384 \mathrm{~m}$.

This refraction coefficient value is quite typical for low passage of the sighting beam over the underlying surface $(1-2 \mathrm{~m})[1,2]$. With the passage of the sighting beam over the channel, the refractive index was positive. It is consistent with the conclusions of [3], where it is established that if the air temperature is higher than the temperature of the water surface layer in a pond, then the vertical angles will be higher than the true angles during most of the day. In our case, the water temperature in the channel was $+28^{\circ} \mathrm{C}$, and the air temperature reached $+33^{\circ} \mathrm{C}$.

The results of the points coordinates' comparison obtained from the results of the tacheometric stroke's placement and from GNSS measurements are presented in Table 2. The points' heights were compared with the results of the geometric leveling class II.

Table 2. Differences of coordinates and heights

\begin{tabular}{|c|c|c|c|}
\hline Point & $\begin{array}{c}\Delta \mathbf{X}=\mathbf{X}_{\text {TRAV }}-\mathbf{X}_{\mathbf{G N S S},}, \\
\text { MM }\end{array}$ & $\begin{array}{c}\Delta \mathbf{Y}=\mathbf{Y}_{\text {TRAV }}-\mathbf{Y}_{\mathbf{G N S S},} \text { MM } \\
\text { TS8 }\end{array}$ initial & $\begin{array}{c}\Delta \mathbf{H}_{\mathbf{H}} \mathbf{H}_{\text {TRIG }}-\mathbf{H}_{\mathbf{L E V}}, \\
\text { MM }\end{array}$ \\
\hline TS2 & initial & initial & -2 \\
\hline TS7 & +39 & +38 & -2 \\
\hline T5 & -3 & +33 & -1 \\
\hline T6 & +5 & +27 & -1 \\
\hline TS6 & $+\mathbf{5 0}$ & $+\mathbf{6 3}$ & -1 \\
\hline T4 & +17 & $+\mathbf{5 4}$ & +4 \\
\hline TS1 & +37 & $+\mathbf{6 9}$ & +1 \\
\hline T10 & +13 & +20 & -2 \\
\hline TS5 & +22 & $+\mathbf{7 2}$ & 0 \\
\hline T9 & -8 & +38 & -12 \\
\hline
\end{tabular}

Differences in coordinates obtained by two different methods reach $72 \mathrm{~mm}$, with the largest discrepancies characteristic of Y axis. Based on the passport accuracy of the satellite receiver $3 \mathrm{~mm}+0.5 \mathrm{ppm}$ and taking the distance to the base station $\mathrm{D}=14 \mathrm{~km}$, the expected error in measuring the baseline will be about $20 \mathrm{~mm}$. The standard errors of the points from the tacheometric stroke along $\mathrm{Y}$ axis do not exceed $5 \mathrm{~mm}$. 
Thus, the standard deviation of the coordinate difference along $\mathrm{Y}$ axis will be $\sigma=21$ $\mathrm{mm}$, and the limiting divergence will be $\Delta=2 \sigma=42 \mathrm{~mm}$. So, the difference values in bold in Table 2 exceed the allowable value. Most likely, the differences in the coordinates of more than $40 \mathrm{~mm}$ were due to the following reasons:

- the points' coordinates of 2015 could be determined relative to other points of the state network, rather than in 2017. Unfortunately, there are no reports on the coordinates determination of previously laid points;

- the presence of an active ionosphere at the measurement site. It can be judged that the ionosphere state was calm on September 12, since the glider index of the ionosphere state (K-index) did not exceed 4 after analyzing the data on the ionosphere state, which presented the National Oceanic and Atmospheric Administration (NOAA). However, one should not forget about possible local disturbances of the ionosphere, which can significantly change refractive index. It directly affects the change in the ionospheric delay.

The refractive index in the ionosphere $n f$ for GNSS signals can be modeled using the formula [4]:

$$
\mathrm{n}_{\mathrm{f}}=1+\mathrm{c}_{2} / \mathrm{f}^{2}+\mathrm{c}_{3} / \mathrm{f}^{3}+\mathrm{c}_{4} / \mathrm{f}^{4}+\ldots
$$

where $\mathrm{c}_{2}, \mathrm{c}_{3}, \mathrm{c}_{4}$ are coefficients depending on $N$ is a number of free electrons in the air volume of $1 \mathrm{~m}^{3}$ along the path of the beam, $f$ is frequency of the electromagnetic signal.

The points' heights obtained from trigonometric leveling were compared with the data of geometric leveling class II. The differences on average were 1-2 $\mathrm{mm}$. This indicated the compensation of the refractive influence in the bilateral (reciprocal) trigonometric leveling.

Table 3 presents the differences in network distances obtained by two methods. The distances measured directly between points differ from satellite definitions by up to $83 \mathrm{~mm}$.

Table 3. The points' differences.

\begin{tabular}{|c|c|c|c|}
\hline Point & Strav. m & SGPS. m & $\Delta \mathbf{S}=\mathbf{S}_{\text {TRAV }}-\mathbf{S}_{\text {GPS. }} \mathrm{mm}$ \\
\hline TS $8-\mathrm{TS} 2$ & 117.5091 & 117.512 & -2.9 \\
\hline TS2 - TS7 & $283, .6012 *$ & 283,605 & -3.8 \\
\hline TS7 - T5 & 91.2946 & 91,239 & 55.6 \\
\hline TS7 - T6 & $179.1049^{*}$ & 179,119 & -14.1 \\
\hline TS7 - T4 & $658.8569^{*}$ & 658,925 & -68.1 \\
\hline $\mathrm{T} 4-\mathrm{TS} 1$ & $370.6051^{*}$ & 370.586 & 19.1 \\
\hline $\mathrm{TS} 1-\mathrm{T} 10$ & 280.6703 & 280.653 & 17.3 \\
\hline T10 - TS5 & 153.2098 & 153.259 & -49.2 \\
\hline TS5 - T9 & 263.2910 & 263.208 & 83.0 \\
\hline T9 - TS8 & $335.9389 *$ & 335.998 & -59.1 \\
\hline TS8 - TS5 & 559.6952 & 559.705 & -9.8 \\
\hline
\end{tabular}


Obviously, differences in distances of more than 30-40 mm are unacceptable, since they are several times higher than the accuracy of measuring distances with a total station $(1.5 \mathrm{~mm}+2.0 \mathrm{ppm})$. The use of the beam method for the works with satellite equipment does not provide adequate measurement control.

\section{Discussion}

A tachometer stroke of $2.5 \mathrm{~km}$ long was laid for the purpose of randomly checking the coordinates and heights of newly laid points. The stroke was not laid at the most favorable time for optical measurements (in the daytime), in a tropical climate. Refraction and turbulent oscillations of the image made it difficult to accurately point the mini-reflector (fig.4).

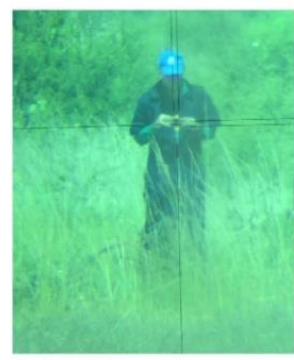

$130 \mathrm{~m}$

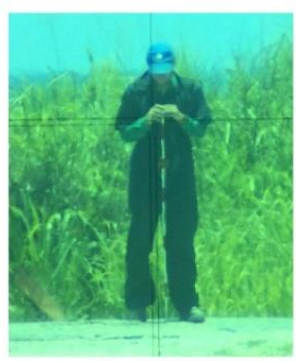

$215 \mathrm{~m}$

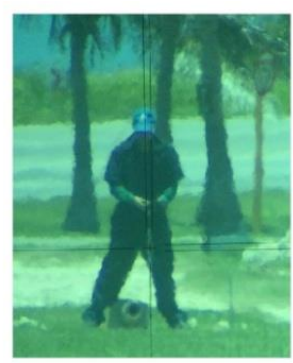

$280 \mathrm{~m}$

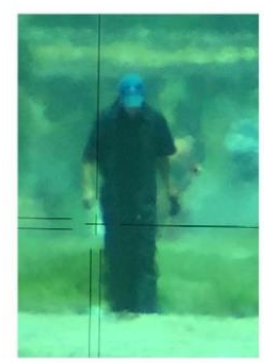

$380 \mathrm{~m}$

Fig. 4. The picture of the reflector through the eyepiece of the total station at different distances.

Conventionally, the quality of the image, depending on the distance, can be divided into the following three groups: good (up to $230 \mathrm{~m}$ ), satisfactory (200-300 m) and unsatisfactory $(300-380 \mathrm{~m})$. Over $400 \mathrm{~m}$ sighting on a mini-reflector is hampered by strong blurring and image oscillation. The horizontal angles measurement is easier to perform, since the guidance is performed on a pole about $1 \mathrm{~m}$ long, which is relatively clearly visible even at extreme distances (up to $380 \mathrm{~m}$ ). Pointing to a mini-reflector during the process of tilt angles measuring is more difficult and at a distance of over $300 \mathrm{~m}$ is not recommended.

The accuracy of the tacheometric stroke could be assessed as high, despite measurements in the hot climate conditions, with refraction influence. The mean square error $(1 \sigma)$ of the weak point did not exceed $12.5 \mathrm{~mm}$. The result of trigonometric leveling should be recognized as very accurate: the maximum difference from the data of II class geometric stroke was $4 \mathrm{~mm}$ (for the TS1 point remote from the original one by $1.2 \mathrm{~km}$ ).

\section{Conclusions}

The geodesic basis on the territory of area objects should be created with GNSS receivers using the network method. At least three baselines should be measured from adjacent points to the next one. The use of the ray method (determination of unknown points' coordinates from a single source) does not have sufficient control and can lead to errors exceeding the passport accuracy of GNSS equipment.

Also, the geodetic basis can be created in the form of a tacheometric moves' system. The experience shows that, a very high accuracy of measurements in the plan is ensured with careful measurements $(\sigma=12.5 \mathrm{~mm}$ for a point remote from the source by $1.2 \mathrm{~km})$.

It is possible to use a combination of two methods: to create a frame network using the (GNSS) network method, and to determine the remaining points by laying tacheometric moves. 
The method should use the same points of the state geodetic network as the source to eliminate the source data errors' effect on the accuracy of the relative position of the points being determined in the process of points' network is concentrated on the territory of a GNSS object.

The use of non-simultaneous two-sided trigonometric leveling at distances of up to 300 $\mathrm{m}$ makes it possible to achieve high accuracy in the heights of points determining, even in tropical climates. The greatest deviation of the point height from the geometric leveling data was $4 \mathrm{~mm}$ with a stroke length of $2.5 \mathrm{~km}$ (on average, the differences were $1-2 \mathrm{~mm}$ ).

\section{References}

1. A.V. Nikonov, Geodesy and aerophotosurveying 1, 28-34 (2014)

2. M.K. Drok, Geodesic series 6, 154-182 (1961)

3. D.D. McCarthy, G. Petit, IERS Conventions (Central Bureau of IERS, Frankfurt and Main, 2010)

4. G.A. Ustavich, G.G. Kitaev, A.V. Nikonov, V.G. Salnikov, Geodesy and aerophotosurveying 4/C, 48-54 (2013) 